Article

\title{
Targeted Polymer-Based Probes for Fluorescence Guided Visualization and Potential Surgery of EGFR-Positive Head-and-Neck Tumors
}

\author{
Robert Pola ${ }^{1}$, Eliška Böhmová ${ }^{1}$, Marcela Filipová ${ }^{1}$, Michal Pechar ${ }^{1}$, Jan Pankrác ${ }^{2}$, \\ David Větvička $^{3}$, Tomáš Olejár $^{3}{ }^{(1)}$, Martina Kabešová $^{3}$, Pavla Poučková $^{3}$, Luděk Šefc $^{2}$, \\ Michal Zábrodský ${ }^{4}$, Olga Janoušková ${ }^{1}$, Jan Bouček ${ }^{4, *}$ and Tomáš Etrych ${ }^{1, *}$ \\ 1 Institute of Macromolecular Chemistry, Czech Academy of Sciences, Heyrovského sq. 2, \\ 16206 Prague 6, Czech Republic; pola@imc.cas.cz (R.P.); bohmova@imc.cas.cz (E.B.); \\ filipova@imc.cas.cz (M.F.); pechar@imc.cas.cz (M.P.); janouskova@imc.cas.cz (O.J.) \\ 2 Center for Advanced Preclinical Imaging (CAPI), First Faculty of Medicine, Charles University, \\ U Nemocnice 5, 12000 Prague 2, Czech Republic; Jan.Pankrac@lf1.cuni.cz (J.P.); sefc@cesnet.cz (L.Š.) \\ 3 Institute of Biophysics and Informatics, First Faculty of Medicine, Charles University, Salmovská 1, \\ 12000 Prague 2, Czech Republic; david.vetvicka@gmail.com (D.V.); tomas.olejar@seznam.cz (T.O.); \\ martina.kabesova@lf1.cuni.cz (M.K.); pavla.pouckova@lf1.cuni.cz (P.P.) \\ 4 Department of Otorhinolaryngology Head and Neck Surgery, First Faculty of Medicine, Charles University \\ and University Hospital Motol, V Úvalu 84, 15006 Prague 5, Czech Republic; michal.zabrodsky@fnmotol.cz \\ * Correspondence: Jan.Boucek@fnmotol.cz (J.B.); etrych@imc.cas.cz (T.E.)
}

Received: 5 November 2019; Accepted: 19 December 2019; Published: 1 January 2020

\begin{abstract}
This report describes the design, synthesis and evaluation of tumor-targeted polymer probes to visualize epidermal growth factor receptor (EGFR)-positive malignant tumors for successful resection via fluorescence guided endoscopic surgery. Fluorescent polymer probes of various molecular weights enabling passive accumulation in tumors via enhanced permeability and retention were prepared and evaluated, showing an optimal molecular weight of 200,000 g/mol for passive tumor targeting. Moreover, poly(N-(2-hydroxypropyl)methacrylamide)-based copolymers labeled with fluorescent dyes were targeted with the EGFR-binding oligopeptide GE-11 (YHWYGYTPQNVI), human EGF or anti-EGFR monoclonal antibody cetuximab were all able to actively target the surface of EGFR-positive tumor cells. Nanoprobes targeted with GE-11 and cetuximab showed the best targeting profile but differed in their tumor accumulation kinetics. Cetuximab increased tumor accumulation after $15 \mathrm{~min}$, whereas GE 11 needed at least $4 \mathrm{~h}$. Interestingly, after $4 \mathrm{~h}$, there were no significant differences in tumor targeting, indicating the potential of oligopeptide targeting for fluorescence-navigated surgery. In conclusion, fluorescent polymer probes targeted by oligopeptide GE-11 or whole antibody are excellent tools for surgical navigation during oncological surgery of head and neck squamous cell carcinoma, due to their relatively simple design, synthesis and cost, as well as optimal pharmacokinetics and accumulation in tumors.
\end{abstract}

Keywords: fluorescence; polymeric conjugate; guided surgery; HPMA; tumor; Head and Neck carcinoma

\section{Introduction}

Surgery remains the primary treatment option for most solid malignant tumors [1]. Precise and complete resection of the whole tumor without unnecessary removal of the neighboring healthy tissue is a prerequisite for a successful outcome [2]. Unfortunately, visual distinction between the malignant and healthy tissue using only the surgeon's naked eye is often almost impossible. Therefore, development of a tumor-specific marker that would visualize the tumor margins is highly desirable. 
Radical resection with adequate negative margins is one of the most important factors influencing the prognoses of patients with head and neck squamous cell carcinoma (HNSCC). Traditionally, the safe margin is defined as greater than $5.0 \mathrm{~mm}$, but similar outcomes are possible with a surgical margin of more than 2.2-3.0 $\mathrm{mm} \mathrm{[3,4].}$

Optical imaging could be used for precise identification of the tumor margins. Indeed, narrow-band imaging (NBI) is already used in clinical practice. This horizontal imaging method is based on the contrast between pathological and healthy microvascular architecture [5]. For evaluation of the deeper tissue layers, vertical diagnostic methods, such as confocal or high resolution endomicroscopy [6], could be used.

Recently, a novel strategy to highlight tumor tissue, mainly tumor margins, using actively and passively targeted fluorescent nanoprobes, was described [7]. Intraoperatively, fluorescence intensity could guide the surgeon to resect tumor tissue together with a safe margin. Epidermal growth factor receptor (EGFR) is known to be overexpressed on the cell surface of various types of malignant tumors, including breast and lung adenocarcinomas (40\% of cases) [8,9], anal cancers [10], glioblastoma (50\%) [11] and epithelial tumors of the head and neck (80-100\%) [12]. Recently, we reported $\mathrm{N}$-(2-hydroxypropyl)methacrylamide (HPMA)-based polymer probes for fluorescence-guided surgery targeted to EGFR with the oligopeptide YHWYGYTPQNVI (GE-11) [13]. It was demonstrated that these EGFR-targeted polymer nanoprobes accumulated to a higher extent in EGFR-positive cells in vitro when compared to the non-targeted control polymer. Similarly, EGFR-targeted nanoprobes were significantly accumulated in EGFR-positive tumors in vivo, thus showing the future potential of these polymer nanoprobes within fluorescence-navigated surgery. Moreover, the EGFR-targeted polymer nanoprobe demonstrated a significant signal in the margin of the tumors, thus clearly showing the benefit of the nanoprobe for the precise navigation of surgeons during solid-tumor removal.

The present study compared several HPMA-based fluorescent probes for EGFR-specific labeling of hypopharyngeal carcinoma cells (FaDu) and breast adenocarcinoma cells (MDA-MB-231) [14,15]. Besides the already mentioned GE-11 targeting oligopeptide designed, prepared and evaluated, polymer probes were also targeted with human epidermal growth factor (EGF), which is the natural ligand for EGFR, and with a monoclonal antibody cetuximab, which is a clinically approved EGFR inhibitor distributed under the trade name Erbitux. EGFR-specific cell binding of all the targeted polymer probes in vitro was evaluated using flow cytometry; fluorescent visualization of EGFR-positive tumors in vivo was performed using an in vivo imaging system in-Vivo Xtreme (Bruker).

In parallel with the actively targeted polymer probes, we also investigated high molecular weight fluorescent polymers without the targeting ligands described above. These polymers passively accumulate within the tumor tissue due to the so-called enhanced permeability and retention (EPR) effect $[16,17]$, which is based on a leaky neovasculature and impaired or missing lymphatic drainage in the tumor.

The potential of the actively and passively targeted polymer probes for the fluorescence-guided surgery of malignant tumors was compared and discussed. Targeted polymeric fluorescent nanoprobes have the potential to increase the safety of oncological surgery in the upper aerodigestive tract and to improve the overall prognosis of patients.

\section{Materials and Methods}

\subsection{Materials}

1-Aminopropan-2-ol, 2,2' -azobis-(isobutyronitrile) (AIBN), $N, N^{\prime}$-diisopropylcarbodiimide (DIC), $N, N$-dimethylacetamide (DMA), N,N-dimethylformamide (DMF), ethyldiisopropylamine (DIPEA), dithiothreitol (DTT), 1-hydroxybenzotriazole (HOBt), methacryloyl chloride, piperidine, trifluoroacetic acid (TFA), triisopropylsilane (TIPS), and all other reagents and solvents were purchased from Sigma-Aldrich (Prague, Czech Republic). TentaGel Rink amide resin, ethyl cyano(hydroxyimino)acetate (Oxyma),

(benzotriazol-1-yloxy)-trispyrrolidinophosphoniumhexafluorophosphate 
(PyBOP), 9-fluorenylmethoxycarbonyl (Fmoc)-amino acid derivatives and 1-(9-fluorenylmethyloxycarbonyl)amino-3,6,9,12,15,18,21,24,27,30,33,36-dodecaoxanonatriacontan-39-oic acid (Fmoc-Peg12-COOH) were purchased from Iris Biotech GmbH (Marktredwitz, Germany). 5-Azidopentanoic acid was obtained from Bachem (Bubendorf, Schwitzerland) and amino-1-(11,12-didehydrodibenzo[b,f]azocin-5(6H)-yl)propan-1-one (Dbco-NH2) was purchased from Click Chemistry Tools (Scottsdale, AZ, USA). Amino and NHS-ester derivatives of fluorescent dyes Cyanine 7 (Cy7-NH2, Cy7-NHS) and Dyomics-633 (Dy-633-NH2, Dy-633-NHS) were obtained from Lumiprobe GmbH (Hannover, Germany) and Dyomics GmbH (Jena, Germany). All amino acids were L-configuration. Human EGF (hEGF) was purchased from Biovision Inc. (Milpitas, CA, USA). Methacryloyl chloride, 1-aminopropan-2-ol, and dichloromethane were distilled immediately before use. All chemicals and solvents were of analytical grade. Solvents were purified and dried using standard procedures.

\subsection{Physico-Chemical Characterization}

Monitoring of the peptide purity and conjugation of the peptide to the reactive copolymer were performed by high-performance liquid chromatography (HPLC) using a Chromolith Performance RP-18e column (100 × $4.6 \mathrm{~mm}$, Merck, Gernsheim, Germany), and a linear gradient of water-acetonitrile, $0 \%-100 \%$ acetonitrile, in the presence of $0.1 \%$ TFA with a UV-vis diode array detector (Shimadzu, Kyoto, Japan). Determination of the molecular weight and dispersity of the copolymers was performed by size exclusion chromatography (SEC) on a HPLC system (Shimadzu, Kyoto, Japan) equipped with refractive index, UV, and multiangle light scattering DAWN 8 EOS (Wyatt Technology Corp., Santa Barbara, CA, USA) detector using a TSK 3000 SWXL column (Tosoh Bioscience, Kyoto, Japan) and $80 \%$ methanol, 20\% $0.3 \mathrm{M}$ acetate buffer $\mathrm{pH} 6.5$ at a flow rate of $0.5 \mathrm{~mL} / \mathrm{min}$. The calculation of molecular weights from the light scattering detector was based on the known injected mass assuming $100 \%$ mass recovery. The molecular weight and dispersity values of the fluorescently labeled polymers were calculated from SEC chromatograms based on pHPMA calibration, using RI detector data. The content of thiazolidine-2-thione (TT) groups was determined spectrophotometrically on a Helios Alpha UV/vis spectrophotometer (Thermo Fisher Scientific, Waltham, MA, USA) using the molar absorption coefficient for TT in methanol, $\varepsilon 305=10,280 \mathrm{~L} \mathrm{~mol}^{-1} \mathrm{~cm}^{-1}$. Determination of dyes Dy-633 or Cy7 was also determined spectrophotometrically using the molar absorption coefficient for Dy-633 (Cy7) in methanol, $\varepsilon 637=159,000 \mathrm{~L} \mathrm{~mol}^{-1} \mathrm{~cm}^{-1}\left(\varepsilon 750=199,000 \mathrm{~L} \mathrm{~mol}^{-1} \mathrm{~cm}^{-1}\right)$.

\subsection{Synthesis of Monomers and Polymer Precursors}

Monomers and amino-reactive copolymer precursor poly(HPMA-co-Ma- $\beta$-Ala-TT) (P-TT) were prepared as described previously [13]. Reactive polymer with dye Dy-633-NH2 or with Cy7-NH2 and control polymers with the dye and without targeting peptides (P-Dy-633 and P-Cy7) were prepared as described previously [13].

\subsection{Synthesis of Peptide-Targeted Nanoprobes}

The peptide-targeted nanoprobes were prepared as described earlier [13] to form the polymer conjugates P-GE11-Dy-633 or P-GE11-Cy7. Similarly, the scrambled control was prepared to form the conjugate P-scrGE11-Cy7 [13]. The amino acid analysis was used for the oligopeptide content determination.

\subsection{Synthesis of hEGF-Targeted Nanoprobes}

HEGF ( $2 \mathrm{mg}$ ) was added to $0.5 \mathrm{~mL}$ of $0.03 \mathrm{M}$ phosphate buffer $\mathrm{pH} 8$ to form a suspension and $4 \mathrm{mg}$ of the polymer precursor P-TT-Dy-633 or P-TT-Cy7 was dissolved in $80 \mu \mathrm{L}$ of DMA. The solution was added dropwise to the suspension of hEGF and vortexed overnight. The next day, the solution was clear, and the completeness of the reaction was verified by HPLC. The reaction mixture was chromatographed on Sephadex G 25 resin in water (PD 10 column, GE Healthcare). The polymeric 
fraction was freeze-dried. The molecular characteristics of conjugates P-EGF-Dy-633 and P-EGF-Cy7 are shown in Table 1. The amino acid analysis was used for the peptide content determination.

\subsection{Synthesis of Monoclonal Antibody (mAb)-Targeted Nanoprobe}

Radical copolymerization in dimethylsolfoxide (DMSO) was applied for the synthesis of semitelechelic polymer precursor (polymer sP-TT, Table 1) containing Boc-protected hydrazide groups. Functionalized initiator 3,3'-[azobis(4-cyano-4-methyl-1-oxobutane-4,1-diyl)] bis(thiazolidine-2-thione) was employed as described earlier [18]. The end-chain reactive maleimide (MI) group was introduced into copolymer sP-TT by the reaction of TT group with $\mathrm{N}$-(2-aminoethyl)maleimide, as described previously [19].

DY-633 and Cy7 containing polymers, sP-DY-633-MI and sP-Cy7-MI, were prepared from sP-MI by the reactions of the hydrazide groups of the polymeric precursor with NHS-esters of the corresponding dyes. Briefly, the polymer precursor sP-MI (50 mg) and DY-633-NHS ester ( $2 \mathrm{mg}, 2 \mu \mathrm{mol})$ were dissolved in $0.5 \mathrm{~mL}$ of DMA and the solutions were mixed together. The reaction was monitored by TLC (eluent: methanol:ethyl acetate:acetic acid 10:4:0.5): RfDY-633-NHS ester $=0.84$, Rfpolymer $=0$. After $2 \mathrm{~h}$ at room temperature, TLC showed ca 85\% yield and the low-molecular weight impurities were removed by gel filtration (Sephadex LH-20, solvent methanol). The purified polymer probe was isolated by precipitation in ethyl acetate. The mAb targeted nanoprobe was prepared in two consecutive steps as described elsewhere [19]. First, the mAb, cetuximab, was mildly reduced by DTT to introduce sulfanyl groups. Then, the reduced $\mathrm{mAb}$ was reacted with semitelechelic copolymers containing MI groups, P-DY-633-MI or P-Cy7-MI, to form the star-like mAb-polymer-dye conjugate. The product was characterized using SEC, UV-VIS spectrophotometry and electrophoresis. The amino acid analysis was used for the mAb content determination.

\subsection{Synthesis of Star Polymers}

The star polymer precursors St-P-1 and St-P-2 were synthesized by grafting the thiazolidine-2-thione (TT)-terminated semitelechelic HPMA copolymer sP-TT onto the 2nd or 4th generation polyamidoamine (PAMAM) dendrimers containing terminal amino groups, as described in [20]. Briefly, semitelechelic copolymer precursor sP-TT with chain-terminated TT groups (32 mg; $0.003 \mathrm{mmol}$ TT groups) was dissolved in $1 \mathrm{~mL}$ of methanol and added into a stirring solution of $0.8 \mathrm{mg}$ of D-NH2 (G2, diaminobutane core, 16 amino groups; $0.002 \mathrm{mmol}$ of dendrimer), or $0.4 \mathrm{mg}$ of D-NH2 (G4, diaminobutane core, 64 amino groups; $0.001 \mathrm{mmol}$ of dendrimer), respectively, in $0.4 \mathrm{~mL}$ of methanol. After $2 \mathrm{~h}$, the reaction was terminated by adding $5 \mu \mathrm{L}$ of 1-aminopropan-2-ol and the remaining free amino groups of the dendrimer were end-capped by reaction with acetic anhydride. The star precursor was freed of low molecular weight impurities by gel filtration (Sephadex LH-20, solvent methanol) and subsequently isolated by precipitation in ethyl acetate. TFA was used for the deprotection of Boc-protected hydrazide groups.

Star polymer conjugates St-P-1-Cy7 and St-P-2-Cy7 (Table 1) bearing fluorescent dye Cy7, attached via a hydrazide bond, were prepared by the reaction of star polymer precursors with Cy7-NHS ester, as described above. Reference polymer sP-Cy7 was prepared from the sP-TT precursor after the removal of TT groups with 1-aminopropan-2-ol, deprotection and attachment of $\mathrm{Cy} 7$ by the procedure described above. 
Table 1. Characteristics of polymer precursors and conjugates.

\begin{tabular}{|c|c|c|c|c|c|c|}
\hline $\begin{array}{l}\text { Polymer Precursors } \\
\text { and Conjugates }\end{array}$ & $M_{\mathrm{n}}$ & $M_{\mathrm{w}} / M_{\mathrm{n}}$ & $\begin{array}{c}\mathrm{TT}^{\mathrm{a}} \text { or Hydrazide } \\
(\mathrm{mol} \%) / \mathrm{mAb} \text { or Peptide } \\
(\mathrm{wt} \%)\end{array}$ & $M_{\mathrm{n}, \mathrm{T}}{ }^{\mathrm{d}}$ & $M_{\mathrm{n}} / M_{\mathrm{n}, \mathrm{T}}^{\mathrm{e}}$ & Dye (wt \%) \\
\hline$P-T T$ & 36,800 & 1.14 & $8.8^{a}$ & - & - & - \\
\hline$s P-T T$ & 15,300 & 1.89 & $5.9^{f}$ & 11,800 & 1.31 & - \\
\hline$s P-M I$ & 19,000 & 1.94 & $5.9^{f}$ & 15,800 & 1.18 & - \\
\hline$s P-C y 7$ & 16,700 & 1.80 & - & - & - & $2.40^{c}$ \\
\hline$s P-D y-633-M I$ & 20,500 & 1.95 & - & 17,500 & 1.17 & $1.12^{b}$ \\
\hline$s P-C y 7-M I$ & 20,500 & 1.95 & - & 18,200 & 1.13 & $1.30^{c}$ \\
\hline$S t-P-1$ & 105,600 & 1.61 & 5.2 & - & - & - \\
\hline St-P-2 & 445,000 & 1.73 & 5.2 & - & - & - \\
\hline P-Dy-633 & 36,800 & 1.14 & - & - & - & $0.80^{\mathrm{b}}$ \\
\hline P-Cy7 & 36,800 & 1.14 & - & - & - & $0.80^{\mathrm{c}}$ \\
\hline P-GE11-Dy-633 & 39,500 & 1.14 & $15.0^{\mathrm{i}}$ & - & - & $0.80^{\mathrm{b}}$ \\
\hline P-GE11-Cy7 & 39,500 & 1.14 & $15.0^{\mathrm{i}}$ & - & - & $0.76^{\mathrm{c}}$ \\
\hline P-scrGE11-Cy7 & 39,500 & 1.14 & $15.0^{\mathrm{i}}$ & - & - & $0.83^{c}$ \\
\hline P-EGF-Dy-633 & 57,000 & 1.28 & $33.3^{g}$ & - & - & $0.64^{\mathrm{b}}$ \\
\hline P-EGF-Cy7 & 57,000 & 1.28 & $33.3^{g}$ & - & - & $0.63^{c}$ \\
\hline mAb-P-Dy-633 & 262,500 & 1.60 & $45.2^{\mathrm{h}}$ & - & - & $0.50^{\mathrm{b}}$ \\
\hline mAb-P-Cy7 & 262,500 & 1.60 & $44.0^{\mathrm{h}}$ & - & - & $0.62^{\mathrm{C}}$ \\
\hline St-P-1-Cy7 & 109,000 & 1.65 & - & - & - & $2.43^{c}$ \\
\hline St-P-2-Cy7 & 457,000 & 1.75 & - & - & - & $2.40^{\mathrm{C}}$ \\
\hline
\end{tabular}

${ }^{a}$ thiazolidine-2-thione content; ${ }^{\mathrm{b}}$ Dy-633content; ${ }^{\mathrm{c}} \mathrm{Cy}-7$ content; ${ }^{\mathrm{d}}$ number-average molecular weight calculated from the TT or maleinimide group content; ${ }^{\mathrm{e}}$ functionality of a polymer; ${ }^{\mathrm{f}}$ content of hydrazide groups (mol\%); $\mathrm{g}$ content of hEGF in conjugate $(w t \%) ;{ }^{h}$ content of monoclonal antibody in conjugate (wt $\left.\%\right) ;{ }^{i}$ content of peptide determinate by aaa; ${ }^{j}$ polymer precursor are listed in upper part using italic style, polymer conjugates are listed in bottom part.

\subsection{Cell Culture}

The cell lines breast carcinoma MDA-MB-231 and HNSC FaDu were purchased from American Type Culture Collection (ATCC, Manassas, VA, USA). MDA-MB-231 was cultured in a mixture of Dulbecco's Modified Eagle Medium (DMEM, Gibco, Gaithersburg, MD, USA) and Roswell Park Memorial Institute (RPMI) 1640 medium (1:1, Gibco), supplemented with 10\% fetal bovine serum (FBS, Sigma-Aldrich, Prague, Czech Republic) and 1\% penicillin-streptomycin (Gibco, Waltham, MA USA). $\mathrm{FaDu}$ cells were grown in Minimum Essential Medium Eagle (Sigma-Aldrich, Prague, Czech Republic), enriched with $2 \mathrm{mM}$ L-glutamine (Gibco, Waltham, MA USA), 1\% ( $v / v)$ non-essential amino acids (NEAA) solution (Gibco, Waltham, MA USA), 10\% FBS (Sigma-Aldrich) and 1\% penicillin-streptomycin (Gibco, Waltham, MA USA). Cell lines were maintained at $37^{\circ} \mathrm{C}$ in a $5 \% \mathrm{CO}_{2}$ humidified atmosphere. FaDu cell line, expressing Red Fluorescent Protein (RFP), was a kind gift from Dr. Trzil (BIOPHARM, Research Institute of Biopharmacy and Veterinary Drugs, Liběchov, Czech Republic). All experiments were conducted on cells in passage less than five.

\subsection{Flow Cytometry}

Cells were harvested in $100 \mathrm{mM}$ HEPES buffer with $20 \mathrm{mM} \mathrm{NaCl}, 10 \mathrm{mM}$ EDTA and $0.5 \%$ bovine serum albumin (BSA, Sigma), pH 7.4 (MDA-MB-231) or 0.05\% trypsin-EDTA (Gibco; FaDu). Collected cells were centrifuged (1500 rpm, $3 \mathrm{~min}$ ) and washed once with PBS containing $0.5 \%$ BSA (PBS-BSA). After washing, $5 \times 10^{4}$ cells/vial were incubated with the appropriate concentration of tested conjugate or phycoerythrin (PE) conjugated anti-EGFR monoclonal antibody (1:10, Exbio, Prague, Czech Republic) for $1 \mathrm{~h}$ at $4{ }^{\circ} \mathrm{C}$ in the dark. Afterwards, cells were washed once from unbounded conjugate with PBS-BSA, centrifuged and mixed with Sytox Blue Dead Cell Stain (Thermo Fisher Scientific, Waltham, MA, USA) to distinguish between live and dead cells. Data acquisition was 
performed using a FACSVerse (Becton Dickinson, Franklin Lakes, NJ, USA) with subsequent analysis using FlowJo software version 10 (Tree Star Inc., Ashland, OR, USA).

\subsection{Statistical Analysis}

All data are presented as mean \pm SEM. The values were determined from at least two experiments performed in duplicate. Saturation binding data were analyzed by GraphPad Prism 5 software, ver. 5.1 (GraphPad Software Inc., San Diego, CA, USA) using non-linear regression curve fit (one-site binding model). Kd values were calculated after subtraction of the control conjugate (non-specific binding), as the targeting conjugate concentration occupies $50 \%$ of the receptors at equilibrium.

For in vivo experiments, one-way ANOVA and a post hoc procedure consisting of multiple two-sided t-tests with the Holm method to control the family-wise error rate were performed using statistical language R. P values lower than 0.05 were considered statistically significant.

\subsection{Immunofluorescence Analysis of Cells}

Cells in microscopic chambers were fixed with buffered $4 \%$ formaldehyde for $15 \mathrm{~min}$ and washed in PBS three times. Subsequently, cells were permeabilized by 0.3\% Triton X-100 in PBS for 15 min and blocked by 5\% BSA in PBS for $30 \mathrm{~min}$. Samples were incubated with primary antibody for $60 \mathrm{~min}$ at ambient temperature, then $60 \mathrm{~min}$ with secondary antibody at ambient temperature. Finally, $25 \mu \mathrm{L}$ of Vectashield ${ }^{\circledR}$ antifade medium with DAPI (Vector Laboratories, Inc., 30 Ingold Road, Burlingame, CA 94010, USA) was added to each cell chamber for nuclear staining.

\subsection{Immunofluorescence Analysis of Tissues}

Tumor tissues obtained from the nude mice or human subjects were fixed with buffered $4 \%$ formaldehyde for $24 \mathrm{~h}$, dehydrated, embedded in paraffin blocks and cut into $5 \mu \mathrm{m}$ slides. Subsequently, the tissue slides were deparaffinized, and rehydrated. Antigen retrieval was performed by boiling in sodium citrate buffer ( $\mathrm{pH}$ 6.0) in a microwave oven for $15 \mathrm{~min}$. Samples were then incubated with primary antibody for $60 \mathrm{~min}$ at ambient temperature and with secondary antibody for $60 \mathrm{~min}$. A droplet of Vectashield ${ }^{\circledR}$ antifade medium with DAPI (Vector Laboratories, Inc., 30 Ingold Road, Burlingame, CA, USA) was added per slide for nuclear staining before mounting, as per manufacturer's protocol.

\subsection{Antibodies}

Primary antibodies anti-EGFR rabbit polyclonal antibody (PA11110, Thermo Fisher Scientific, Pierce Biotechnology, Rockford, IL, USA), dilution 1:200, and anti-human cytokeratin mouse monoclonal antibody AE1/AE3 (M3515, DAKO Denmark A/S, Glostrup, Denmark), dilution 1:200. Secondary antibodies donkey anti-mouse IgG $(\mathrm{H}+\mathrm{L})$ highly cross-adsorbed secondary antibody, Alexa Fluor 680 (A10038, Invitrogen, Paisley, UK), dilution 1:1000 and donkey anti-rabbit IgG (H+L) highly cross-adsorbed secondary antibody, Alexa Fluor 488 (A-21206, Invitrogen), dilution 1:1000.

\subsection{Fluorescence Microscopy}

The confocal Leica TCS SP5 microscope (Leica Microsystems, Wetzlar, Germany) was used for imaging. Alexa Fluor 488 was excited using $488 \mathrm{~nm}$ argon laser line, Alexa Fluor 680 was excited using $633 \mathrm{~nm}$ HeNe laser line. DAPI was excited using $405 \mathrm{~nm}$ laser. Open source FIJI ImageJ was utilized for the processing of obtained images [21].

\subsection{Intravital Tumor Accumulation Assessment}

All animal experiments were performed in accordance with the Act on Experimental Work with Animals (Public Notice of the Ministry of Agriculture of the Czech Republic No. 246/1992, No. 311/1997, No. 207/2004; Decree of the Ministry of the Environment of the Czech Republic No. 117/1987; and Act of the Czech National Assembly No. 149/2004) of the Czech Republic, which is fully compatible with 
the corresponding European Union directives. Animal study protocol, covering all hereafter described animal experiments, was approved by the Czech Ministry of Education Youth and Sports (Approval No. MSMT-12181/2016-4, date of approval: 15 May 2016).

All patients signed informed consent before entering the study and the study protocol was approved by the Central Ethics Committee of Hospital Motol, Prague, Czech Republic (Approval No. AZV16-28594A, date of approval: 24 June 2015). In addition, all data were analyzed with respect to patient privacy.

FaDu-RFP cells, where RFP means red fluorescent protein, were trypsinized, spun and

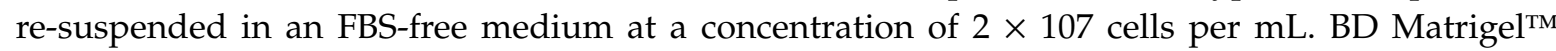
(I.T.A.-Intertact, Ltd., Prague, Czech Republic) was added (1/2 of the cell suspension volume) and $0.15 \mathrm{~mL}$ of the mixture was subcutaneously administered $(2 \times 106$ cells per mouse) into the abdominal right flank of athymic nude mice (Velaz, Ltd. and Charles River Laboratories International, Inc., Prague, Czech Republic). When the tumors had reached a minimum size of $6 \mathrm{~mm}$ in diameter, mice were divided into groups of eight and randomly allocated for individual intravenous conjugate administration. In case of conjugates sP-Cy7, St-P-1-Cy7 and St-P-2-Cy7, $0.25 \mathrm{mg}$ of probes per mouse were administered, dissolved in $0.1 \mathrm{~mL}$. For the experiment with actively targeted conjugates mAb-P-Cy7, P-scrGE11-Cy7, P-GE11-Cy7 and P-EGF-Cy7, the amount of conjugates was unified based on the Cy7 dye content, as these conjugates had uneven $\mathrm{Cy} 7$ content. Tumor accumulation and the biodistribution of polymeric carriers conjugated to Cy7 were determined using the Xtreme In Vivo Imaging System (Bruker BioSpin, Ettlingen, Germany) by intravital imaging $15 \mathrm{~min}, 4$ and $24 \mathrm{~h}$ after administration, as described in [13]. Images were taken separately for both RFP and Cy7 fluorescent channel (exposition time of $5 \mathrm{~s}$ for both channels), and the reflectance images were acquired. The light source was Xenon Arc Lamp with $400 \mathrm{~W}$ power. Band filters were used as excitation emission filters to pass a single wavelength of +- about $5 \mathrm{~nm}$. The camera has a CCD sensor and is cooled to $-65^{\circ} \mathrm{C}$. Open source software FIJI was utilized for the adjustment of obtained images and quantification of ROIs [21].

\section{Results}

\subsection{Synthesis of the Polymer Nanoprobes}

Various polymer-based nanoprobes were designed and synthesized, tailoring their potential to visualize tumors to potentiate fluorescence-guided surgery. Linear and star polymer probes and probes targeted with three structurally different EGFR-targeting units, oligopeptide GE-11, EGF and anti-EGFR $\mathrm{mAb}$ cetuximab, were successfully prepared as shown in Table 1 , using free radical polymerization and controlled RAFT radical polymerization. Using the grafting-to approach, star-like polymer carriers were successfully synthesized by controlled grafting of semitelechelic HPMA copolymers to a PAMAM-based dendrimer core. The molecular weight was controlled by selection of the dendrimer generation and the number of the polymer grafts attached to the dendrimer core, producing polymers with a $\mathrm{Mw}$ of 170 and $770 \mathrm{~kg} / \mathrm{mol}$, which, with a linear polymer of $30 \mathrm{~kg} / \mathrm{mol}$, formed the broad molecular weight sample set.

The GE11- and GE11scr-targeted polymers were prepared by the attachment of oligopeptides to the polymer precursor containing TT groups via an aminolytic reaction. The binding in dry organic solvent led to the complete attachment of oligopeptides; the final nanoprobes contained $15 \% \mathrm{wt}$. of the oligopeptide, corresponding to 5-6 oligopeptides attached to each polymer chain. A similar reaction was also employed for the preparation of EGF-targeted nanoprobes in an aqueous buffer at $\mathrm{pH} 8$, which enabled the partial deprotonation of amino groups leading to the aminolytic reaction with TT groups of the polymer precursors. The partial deprotonation was chosen to minimize possible cross-linking resulting from the reaction of multiple amino groups of EGF with polymer precursors. The GPC chromatograms showed that the polymers were not significantly cross-linked, and their molecular weight is equal to the attachment of approximately two hEGF molecules per one polymer chain. Finally, a mAb targeted polymer nanoprobe was synthesized by controlled grafting of the partly 
reduced $\mathrm{mAb}$ molecule, enabling eight polymer carriers attached to one $\mathrm{mAb}$. For the schematic description of the syntheses of peptide-targeted polymer systems, see Scheme 1 and Figure S1.

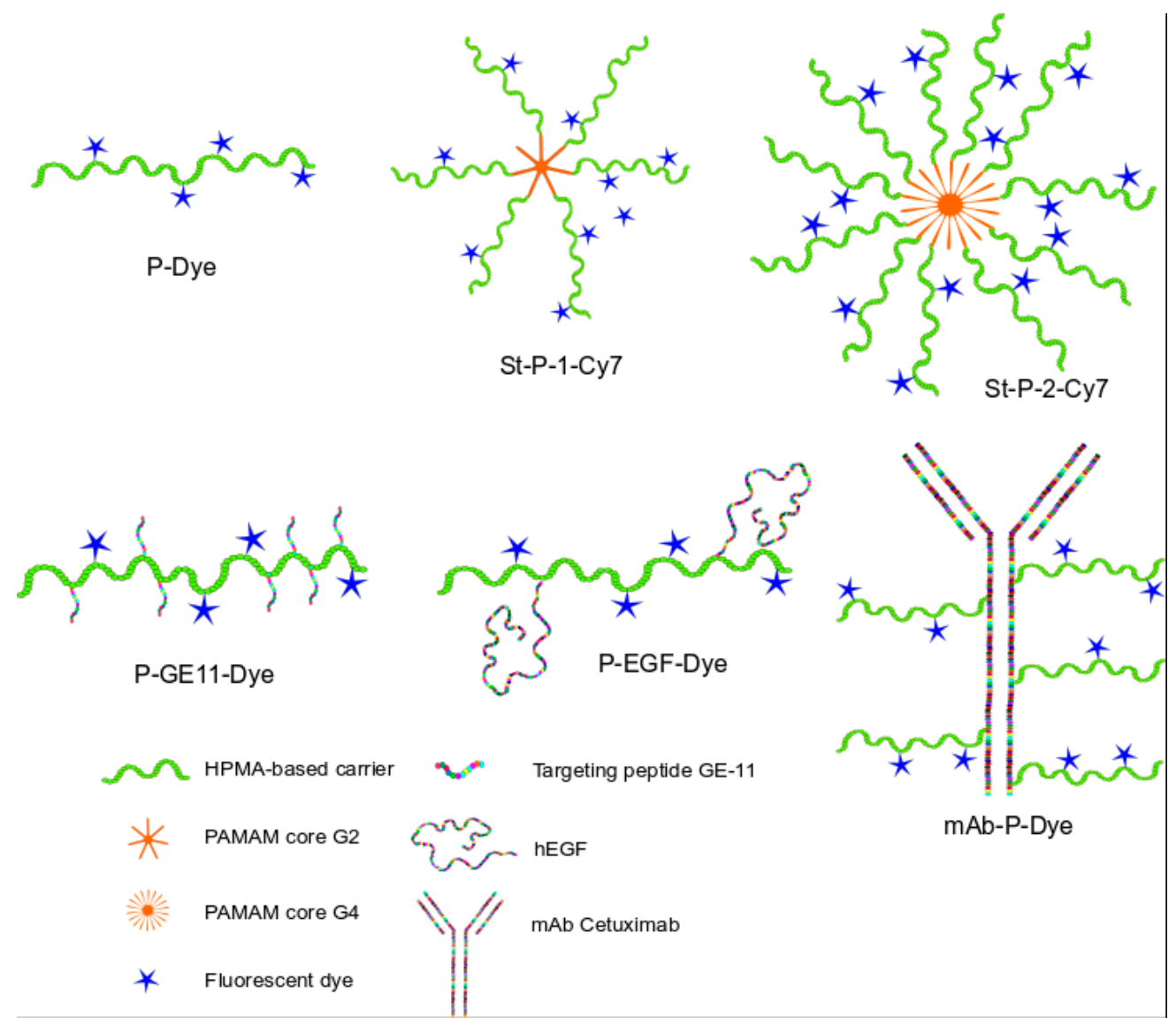

Scheme 1. The structure of prepared polymer nanoprobes.

\subsection{Binding Affinity of Polymeric Conjugates to EGFR-Positive Cells}

To evaluate the binding affinities of prepared polymeric conjugates, first the EGFR expression level was determined in breast cancer cells MDA-MB-231 and hypopharyngeal carcinoma cells FaDu. Flow cytometry analysis revealed that FaDu cells expressed two-times more EGFR than MDA-MB-231 (Figure 1).
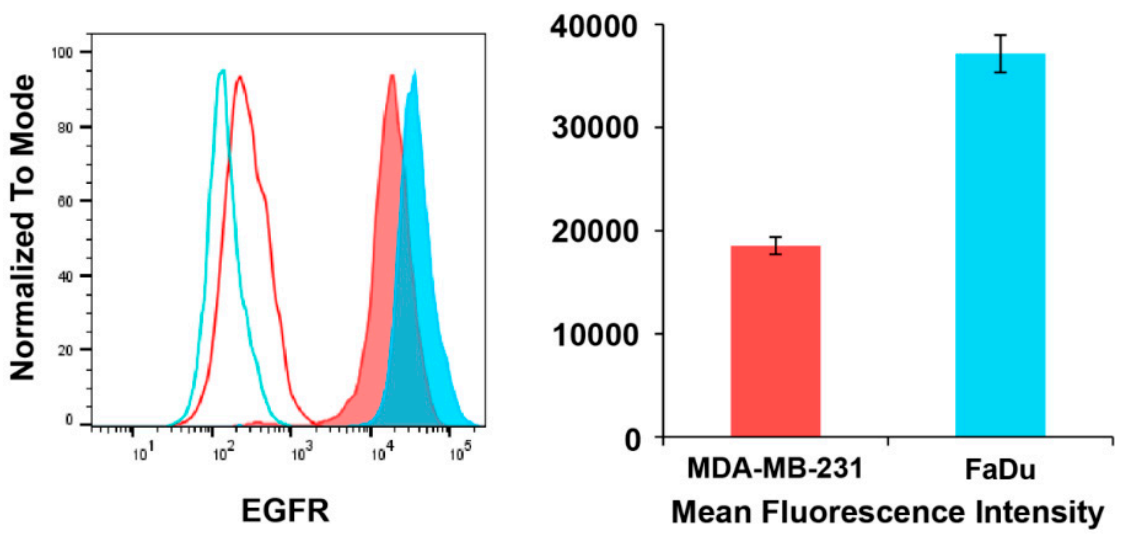

Figure 1. Quantification of epidermal growth-factor receptor (EGFR) expression on MDA-MB-231 (red) and $\mathrm{FaDu}$ (blue) cells by flow cytometry. Expression level of EGFR is shown by the filled histograms. Solid lines represent negative controls. Data are presented as mean \pm SEM of two experiments performed in duplicate. 
Investigation of the targeting ability of prepared conjugates by flow cytometry showed that the mAb-P-Dy-633 conjugate with a Kd of 274 ng/mL (MDA-MB-231) or 591 ng/mL (FaDu) (Figure 2) had the best binding affinity. P-EGF-Dy-633 targeted $50 \%$ of the receptors at equilibrium $(\mathrm{Kd})$ at a concentration of $2.33 \mu \mathrm{g} / \mathrm{mL}$ (MDA-MB-231) and $5.24 \mu \mathrm{g} / \mathrm{mL}$ (FaDu). In the case of P-GE11-Dy-633, increasing concentration of the conjugate increased binding affinity to EGFR in MDA-MB-231, but did not reach saturation, even at the highest concentration of $100 \mu \mathrm{g} / \mathrm{mL}$. In comparison with P-EGF-Dy-633 and mAb-P-Dy-633, the binding affinity of P-GE11-Dy-633 to EGFR in FaDu cells was minimal.

MDA-MB-231

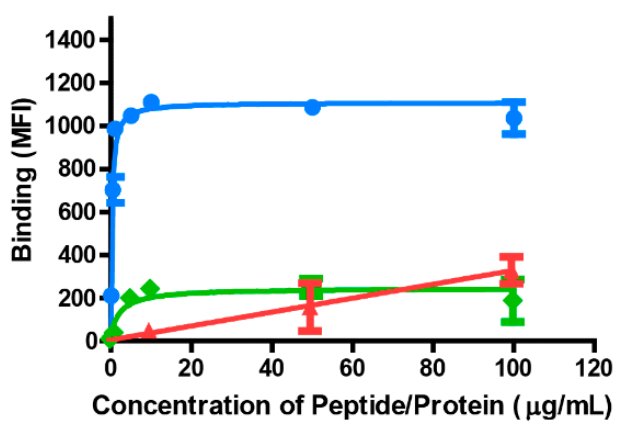

FaDu

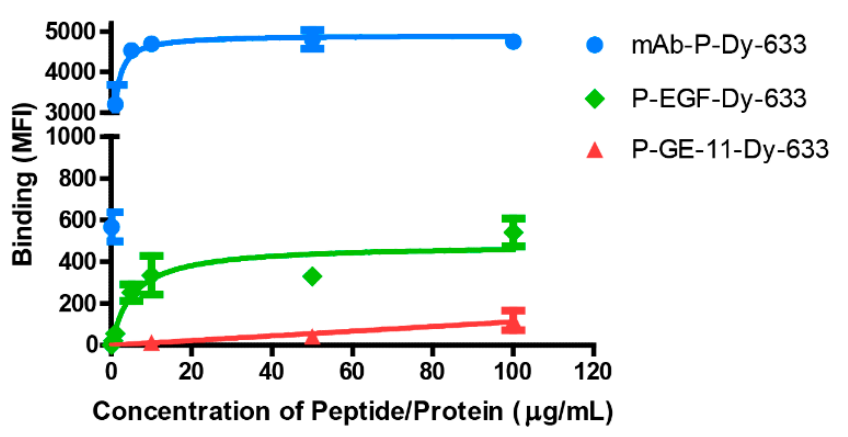

Figure 2. Binding of mAb-P-Dy-633, P-EGF-Dy-633 and P-GE11-Dy-633 conjugates to EGFR expressed in MDA-MB-231 and FaDu cells. Each datapoint represents the mean \pm SEM of at least two experiments performed in duplicate (SEM showed after reaching value > $50 \mathrm{MFI}$ ).

\subsection{EGFR Expression}

As a HNSCC in vivo model, the expression of the target EGFR was evaluated in the human pharyngeal carcinoma cell line FaDu. Expression was determined in vitro cultured cells and mouse xenograft tumor sections. As shown in Figure 3, immunohistochemical staining revealed clear membrane EGFR expression on both FaDu cells and established FaDu tumors. The chosen FaDu HNSCC model is thus appropriate for testing the EGFR-targeted polymeric probes. Moreover, the expression of EGFR by cancer cells in HNSCC tissues was also confirmed (see Figure 3) for 25 independent patient samples. Moreover, the EGFR expression was also validated using Western blot analysis of EGFR expression on FaDu and MDA cells, Figure S2. Indeed, both glycosilated and non-glycosilated variants were found on FaDu cells.

\subsection{Tumor Accumulation Based on Molecular Weight of Conjugates}

To assess the influence of molecular weight on tumor accumulation, three different molecular weights $(26,170$ and $770 \mathrm{~kg} / \mathrm{mol})$ of conjugates were tested after intravenous application at three time points (15 $\mathrm{min}, 4$ and $24 \mathrm{~h}$ after application). In the case of conjugate sP-Cy7 (26 kg/mol), low signal from the tumor site was documented, however, there was no significant increase in the fluorescence signal from the tumor over time (Figure 4). In contrast, higher molecular weight conjugates showed improved tumor accumulation which increased over time. At $4 \mathrm{~h}$ after intravenous administration, the accumulation improved significantly for St-P-1-Cy7 (170 kg/mol) and St-P-2-Cy7 (770 kg/mol), with St-P-1-Cy7 being significantly superior to St-P-2-Cy7 (Figure 4).

Similar results were recorded at $24 \mathrm{~h}$. Fluorescent images of whole mice revealed that the lower molecular weight conjugate sP-Cy7 accumulated in kidneys prior to elimination in urine, while the other two conjugates do not show substantial kidney accumulation based on their increased size (Figure 5A). Tumor accumulation was confirmed by colocalization with the RFP signal from the tumor cells (Figure 5B). 


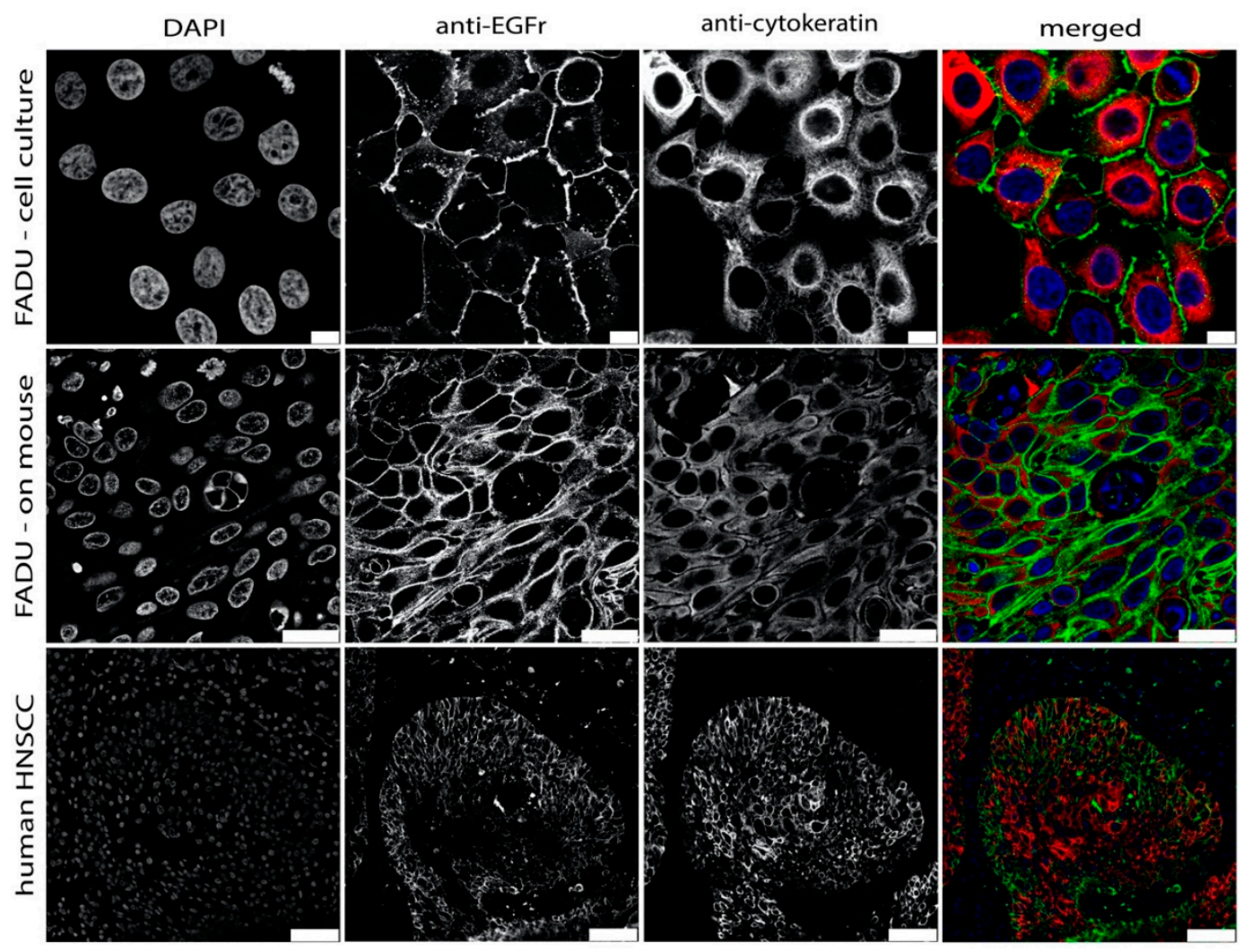

Figure 3. EGFR expression in FaDu cell tissue culture, FaDu transplanted in nude mice and in human poorly differentiated squamous carcinoma. The samples were stained with EGFR antibody (green) and counterstained with an antibody against cytokeratin (red) to confirm the epithelial origin of the tumor (carcinoma), and with DAPI for nuclear stain (blue). Scale bars: FADU cell culture - $10 \mu \mathrm{m}$; FADU on mouse - $25 \mu \mathrm{m}$; human HNSCC - $50 \mu \mathrm{m}$.

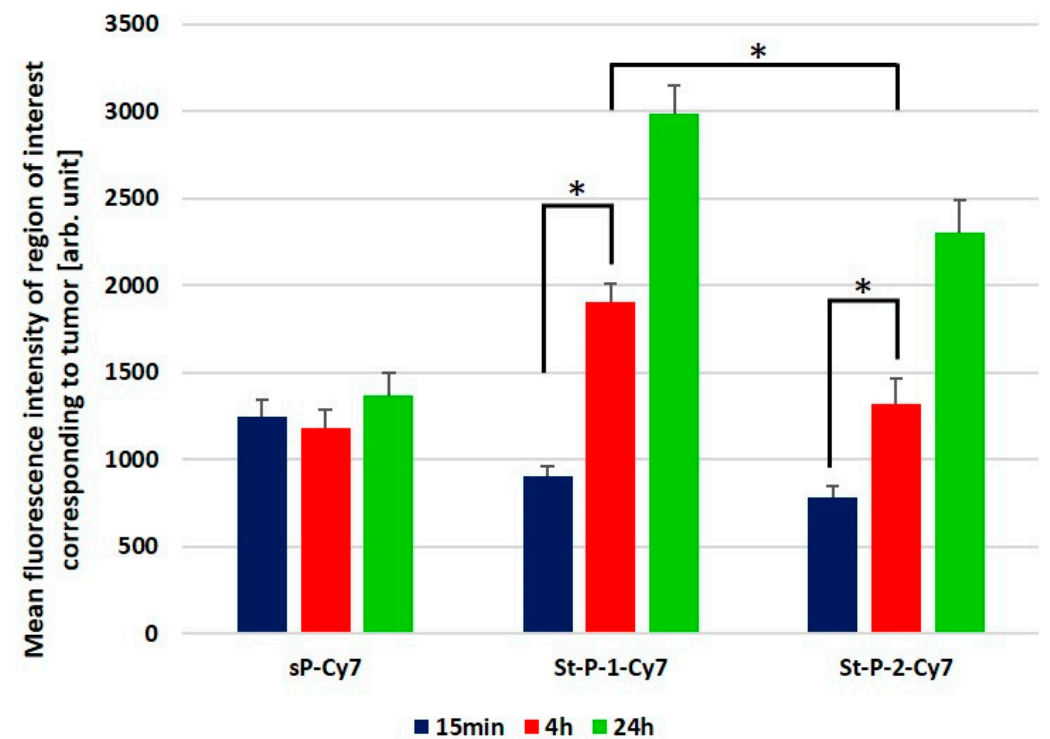

Figure 4. In vivo tumor accumulation of different molecular weight polymer probes (sP-Cy7, St-P-1-Cy7 and St-P-2-Cy7) with the same percent (2.4\%) weight of Cy-7 fluorescent dye. MFI was determined by the In-Vivo Xtreme In Vivo Imaging System. The data are presented as the mean \pm SEM of duplicates from two independent experiments. ${ }^{*} P$ values $<0.001$. Number of mice $n=8$. 
A

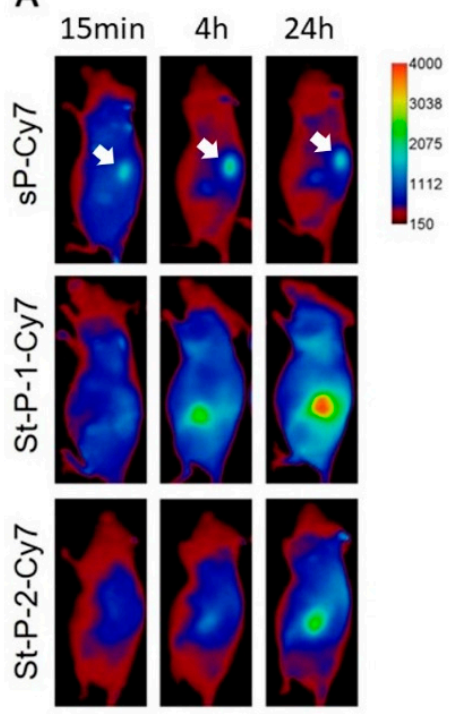

B

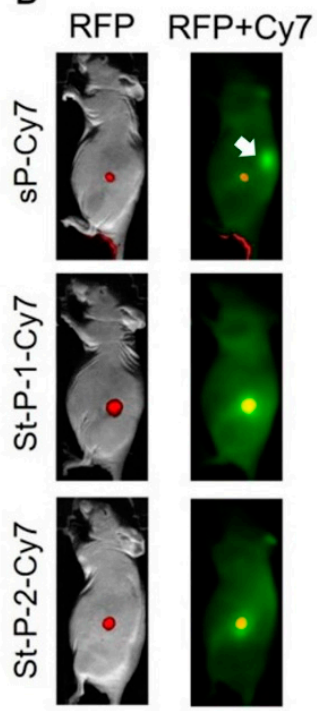

Figure 5. Passive tumor accumulation of different molecular weight polymer probes sP-Cy7, St-P-1-Cy7 and St-P-2-Cy7. White arrow shows kidney. (A) Cy-7 fluorescence signal in representative mice at three different times; (B) RFP signal after $24 \mathrm{~h}$ post injection from red fluorescent protein (RFP)-expressing tumor cells in red and Cy-7 fluorescence signal in green. Number of mice $n=8$.

\subsection{Tumor Accumulation Based on Active Targeting}

Next, the following conjugates actively targeted against EGFR were tested: samples with clinically approved mAb cetuximab (mAb-P-Cy7), human recombinant EGF (P-EGF-Cy7), GE-11 (P-GE11-Cy7) and sP-Cy7 as a non-targeted control. At 4 and $24 \mathrm{~h}$ intervals, the sample with GE-11 (P-GE11-Cy7) exhibited significantly higher accumulation in tumor tissues than in the nontargeted sP-Cy7 conjugate (Figure 6), thus proving the additional value of active oligopeptide targeting.

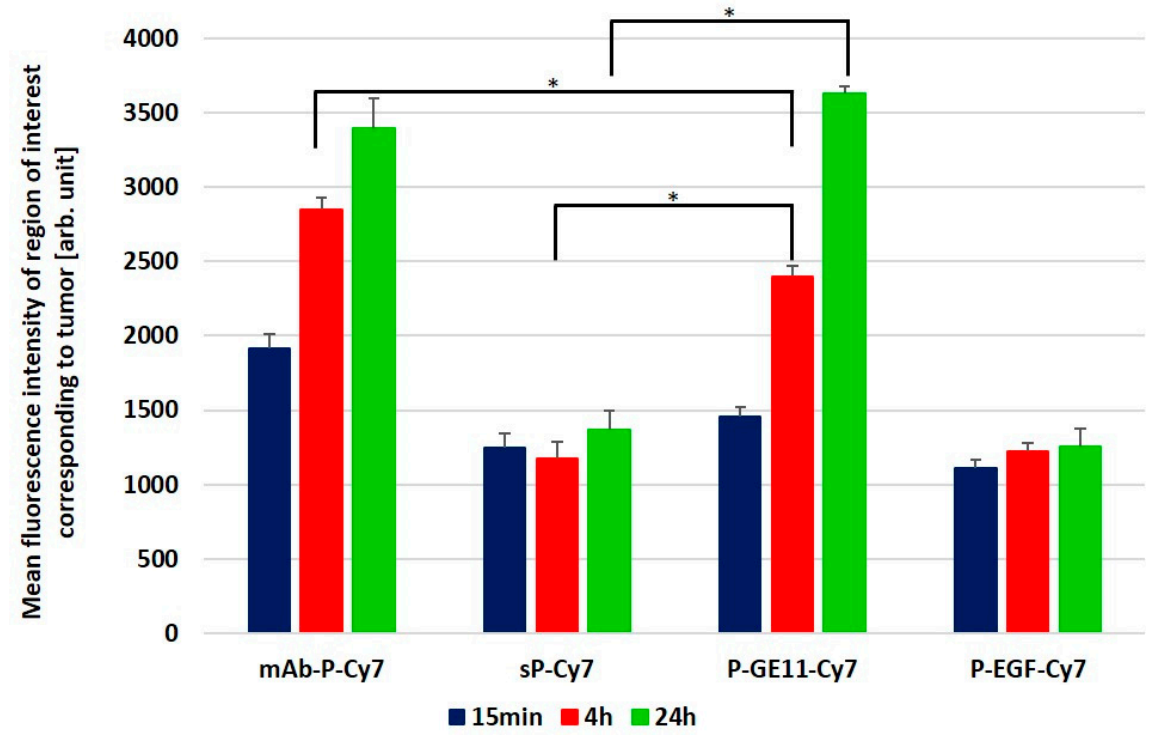

Figure 6. In vivo tumor accumulation of polymer probes containing Erbitux, GE-11, human EGF or no targeting moiety (mAb-P-Cy7, P-GE11-Cy7, P-EGF-Cy7 and sP-Cy7). The MFI was determined by the In-Vivo Xtreme In Vivo Imaging System. The data are presented as the mean \pm SEM of duplicates of two independent experiments. ${ }^{*} P$ values $<0.05$. Number of mice $n=8$.

Figure 7A depicts the distribution over time, revealing the accumulation of samples mAb-P-Cy7, P-scrGE11-Cy7 and P-GE11-Cy7 in the tumor. Efficient tumor accumulation was confirmed by 
colocalization with the RFP signal derived from the RFP-expressing tumor cells (Figure 7B). In addition to successful tumor accumulation, P-GE11-Cy7 also gave a strong signal from kidneys, indicating concurrent renal excretion (Figure 7).

A

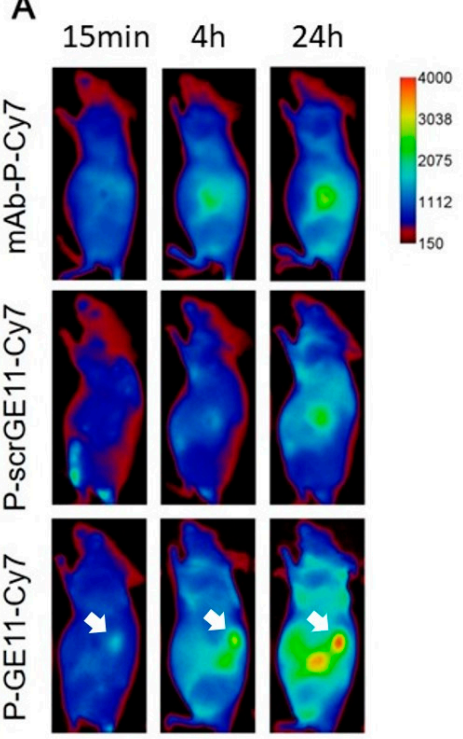

B

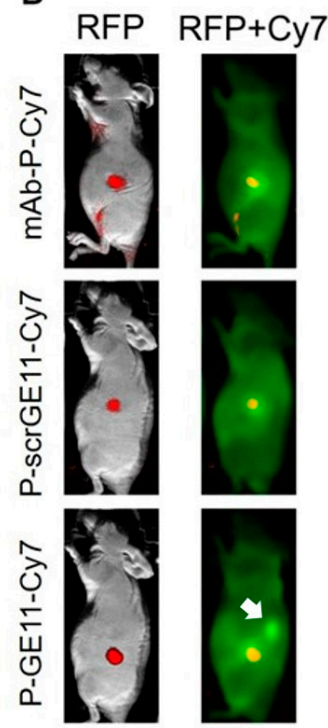

Figure 7. Tumor targeting of polymer probes containing Erbitux, GE-11scr and GE-11 (mAb-P-Cy7, P-scrGE11-Cy7, P-GE11-Cy7). White arrow shows kidney. (A) Cy-7 fluorescence signal in representative mice at three different times; (B) RFP signal from RFP-expressing tumor cells in red and Cy-7 fluorescence signal in green, showing the colocalization of signals. Number of mice $n=8$.

The conjugate with EGF (P-EGF-Cy7) exhibited no significant accumulation in the tumor over time. At all intervals, the strength of the signal was similar to that of the control polymer (sP-Cy7) (Figure 6). In the case of P-EGF-Cy7, analysis of the Cy-7 signal beyond the tumor area revealed strong accumulation of the conjugate in kidneys and liver (Figure 8A). Additionally, poor tumor accumulation resulted in low colocalization with the tumor-related RFP signal (Figure 8B).

A

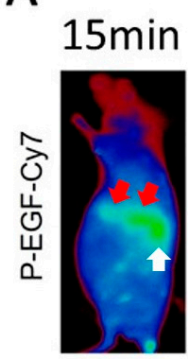

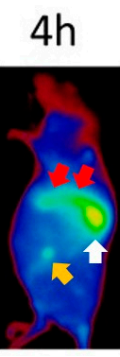
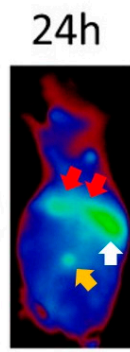

B

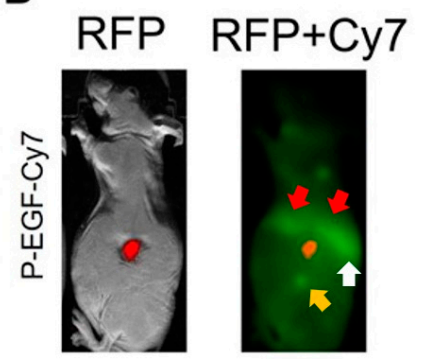

Figure 8. Tumor targeting of polymer probe containing recombinant human EGF (P-EGF-Cy7). White arrow shows kidney, red represents liver and yellow marks the bladder. (A) Cy-7 fluorescence signal in representative mouse at three different times; (B) RFP signal from RFP-expressing tumor cells in red and Cy-7 fluorescence signal in green.

\section{Discussion}

\subsection{Synthesis of the Polymer Nanoprobes}

The free radical and RAFT polymerization techniques were used successfully for polymer nanoprobe synthesis. The RAFT technique resulted in copolymers with a very narrow molecular weight distribution, which exhibit more uniform biodistribution, highly desirable characteristics for 
a good contrast in the fluorescent imaging. Consequently, this should also facilitate the regulatory approval process for eventual clinical application.

The controlled "grafting-to" approach enabled the tailored synthesis of polymer nanoprobes in a broad range of molecular weights, from 26 to $770 \mathrm{~kg} / \mathrm{mol}$. The size of the polymer probes was controlled by selection of the appropriate generation of the PAMAM dendrimer core and by adjustment of the ratio between the polymer and the dendrimer. Similarly, the aminolytic reaction between the reactive TT groups of the polymer precursors and the amino groups of the oligopeptides or EGF under controlled conditions was employed to load a sufficient amount of the EGFR-targeting moieties to the polymer conjugate. For the mAb binding to the polymer, we selected a regio-selective "grafting-to" approach to minimize undesired modification of the binding site of the mAb to EGFR. Thiol groups of $\mathrm{mAb}$, generated upon partial reduction of the disulfide bridges of the protein, were used for a single point attachment of the semitelechelic polymers to $\mathrm{mAb}$.

\subsection{Binding Affinity of Polymeric Conjugates to EGFR-Positive Cells}

The binding affinity of prepared conjugates mAb-P-Dy-633, P-EGF-Dy-633 and P-GE11-Dy-633 was evaluated in vitro using two cell lines, MDA-MB-231 and FaDu. FaDu expressed twice as much EGFR as MDA-MB-231, thus influencing the detected fluorescence intensities. However, the trend in binding affinity was similar in both cell lines, with the highest binding affinity observed for the cetuximab-targeted polymer conjugate mAb-P-Dy-633. The corresponding Kd values for MDA-MB-231 $(0.274 \mu \mathrm{g} / \mathrm{mL})$ and for FaDu cells $(0.591 \mu \mathrm{g} / \mathrm{mL})$ were approximately ten times lower than those observed for P-EGF-Dy-633, (MDA-MB-231 - $2.33 \mu \mathrm{g} / \mathrm{mL}$; FaDu - $5.24 \mu \mathrm{g} / \mathrm{mL}$ ). The results are in agreement with recently published data [22], showing much lower Kd for cetuximab, which can even compete with EGF in binding to EGFR. The two-fold higher calculated $\mathrm{Kd}$ for FaDu (in comparison with MDA-MB-231) is in accordance with the two-fold higher expression of EGFR in FaDu cells, Figure 1.

The polymer conjugate P-GE11-Dy-633 showed the lowest binding affinity. Although GE-11 peptide was previously reported as a targeting ligand for the EGFR [23], in our study, its efficiency to bind to EGFR in vitro was the lowest in comparison to both $\mathrm{mAb}$ (cetuximab) and the EGF. Indeed, recently it was published that GE-11 containing systems are significantly internalized by the FaDu cells [13], thus we can conclude that, despite their low binding affinity, the GE11-containing polymers could be internalized significantly in a short time. In the case of MDA-MB-231 cells, increasing fluorescent intensity with increasing amounts of P-GE11-Dy-633 was observed, with no saturation up to the peptide concentration of $100 \mu \mathrm{g} / \mathrm{mL}$; however, the fluorescent intensity of P-EGF-Dy-633 or $\mathrm{mAB}-\mathrm{P}-\mathrm{Dy}-633$ reached saturation below $10 \mu \mathrm{g} / \mathrm{mL}$ of the protein concentration. Interestingly, when FaDu cells were used, almost no binding affinity of P-GE11-Dy-633 was detected. It is possible that the binding affinity of GE11 to EGFR is variable in different EGFR-positive cell lines, due to the structural variation in EGFR, which does not change the binding affinity of cetuximab and EGF as much as EGFR-binding structures, but is pronounced in the case of GE11, with a much higher Kd for EGFR [24].

\subsection{Tumor Accumulation Based on Molecular Weight of Conjugates Versus Active Targeting}

It seems that nanoprobes with a molecular weight above the renal threshold $(50,000 \mathrm{~g} / \mathrm{mol})$ and below 500,000 g/mol are optimal for passive targeting to solid tumors [25] due to the EPR effect; while sP-Cy7 is rapidly eliminated via kidney and St-P-2-Cy7 is too big for effective tumor accumulation, St-P-1-Cy7 showed a promising accumulation profile. As expected, mAb-P-Cy7 exhibited pronounced accumulation in the tumor after $15 \mathrm{~min}$, thus showing excellent synergism of passive and active tumor targeting. The conjugated antibody provides a higher molecular weight (above the renal threshold for HPMA copolymers), thus prolonging the circulation time and decreasing renal excretion [26]. Likewise, $\mathrm{mAb}-\mathrm{P}-\mathrm{Cy} 7 \mathrm{showed}$ significantly superior tumor accumulation at $15 \mathrm{~min}$ and $4 \mathrm{~h}$ compared with P-GE11-Cy7. At $24 \mathrm{~h}$ P-GE11-Cy7 achieved slightly better results, though the difference was insignificant. The outstanding tumor accumulation of P-GE11-Cy7 in vivo is contradictory to the 
in vitro data presented and discussed above. Indeed, the scrambled GE11-targeted probe did not show any significant difference with respect to the sP-Cy7 accumulation, thus supporting the efficacy of the GE11 oligopeptide for the EGFR targeting in vivo. We hypothesize that passive targeting of P-GE11-Cy7 with a molecular weight close to the limit of the renal filtration is highly elevated and, in combination with the slightly pronounced active EGFR-targeting, leads to the observed tumor accumulation after $24 \mathrm{~h}$. Conversely, the effect of active targeting using $\mathrm{mAb}$ is limited by the saturation of the EGFR receptors, as in vitro. The results proved the potential of targeted polymer nanoprobes, demonstrating their advantages and limitations. To achieve rapid tumor accumulation, the best option is highly specific $\mathrm{mAb}$ targeting, whereas polymer nanoprobe with targeting oligopeptides, relatively simple in design and synthesis, and with a low cost, is acceptable for navigated surgery when a short time between probe application and surgery is not required. Moreover, the GE11-containing polymer probe is easily clearable from the organism, in contrast to a much larger star or mAb-targeted polymer probes, proving the potential applicability of the GE-11 targeted polymer conjugate for tumor visualization. Moreover, we can also conclude that St-P-1-Cy7 has similar accumulation profile to $\mathrm{mAb}-\mathrm{P}-\mathrm{Cy} 7$, thus proving the prevalence of the EPR effect in the case of high molecular weight systems over active targeting.

Interestingly, P-EGF-Cy7 did not exhibit any significant tumor accumulation, while in vitro results showed a relatively strong interaction with EGFR. However, a strong accumulation of P-EGF-Cy7 in liver and kidney was observed at all time intervals, which may be due to the cross-reactivity of the human EGF [27] with murine EGFR overexpressed in liver [28] and kidney of mice [29]. No significant accumulation of GE11 and mAb cetuximab in liver and kidney was observed, thus proving no or very low cross-reactivity with mouse EGFR. Moreover, we have not observed any sign of toxicity after the injection of the polymer probe, e.g., weight loss or other toxicity symptoms. Similarly, polymer probes did not show toxicity when measured on cancer cell lines in vitro [13].

\section{Conclusions}

Tumor-targeted polymer probes, intended for the visualization of EGFR-positive malignant tumors for successful resection via fluorescence-guided endoscopic surgery, were successfully designed, prepared and evaluated. The optimal molecular weight is $200,000 \mathrm{~g} / \mathrm{mol}$ for passive tumor targeting, showing maximal accumulation from $15 \mathrm{~min}$ to $24 \mathrm{~h}$. The nanoprobes targeted with GE-11 and cetuximab exhibited a very good targeting profile but differed in their tumor accumulation kinetics. Cetuximab should be preferentially used for the visualization of tumors after a short time ( $15 \mathrm{~min}$ after injection), while the GE11-targeted nanoprobe is excellent for visualization of tumors more than $4 \mathrm{~h}$ after nanoprobe injection. In conclusion, fluorescent polymer probes targeted with oligopeptide GE11 or with cetuximab are excellent tools for navigation during oncological surgery of HNSCC, due to their relatively simple design, synthesis, low cost, optimal pharmacokinetics and accumulation in tumors.

Supplementary Materials: The following are available online at http://www.mdpi.com/1999-4923/12/1/31/s1, Figure S1: Schematic synthetic overview peptide-targeted polymer probes. Figure S2. Western blot analysis of EGFR expression on FaDu a MDA cells.

Author Contributions: Conceptualization, T.E. and J.B.; synthesis, R.P., E.B. and M.P.; in vitro analysis, M.F., T.O. and O.J.; in vivo analysis, J.P., D.V., M.K., M.Z. and L.Š.; writing—original draft preparation, R.P., T.E., D.V. and J.B.; funding acquisition, P.P., T.E. and J.B. All authors have read and agreed to the published version of the manuscript.

Funding: This work was supported by the Ministry of Health of the Czech Republic (grant 16-28594A).

Conflicts of Interest: The authors declare no conflict of interest.

\section{References}

1. Baddour, H.M. The Importance of Margins in Head and Neck Cancer. J. Surg. Oncol. 2016, 113, $248-255$. [CrossRef] [PubMed]

2. Kamat, M. A comprehensive review of surgical margin in oral squamous cell carcinoma highlighting the significance of tumor-free surgical margins. J. Cancer Res. Ther. 2019, 15, 449-454. [CrossRef] [PubMed] 
3. Zanoni, D.K.; Migliacci, J.C.; Xu, B.; Katabi, N.; Montero, P.H.; Ganly, I.; Shah, J.P.; Wong, R.J.; Ghossein, R.A.; Patel, S.G. A Proposal to Redefine Close Surgical Margins in Squamous Cell Carcinoma of the Oral Tongue. JAMA Otolaryngol. Neck Surg. 2017, 143, 555. [CrossRef]

4. Dik, E.A.; Willems, S.M.; Ipenburg, N.A.; Adriaansens, S.O.; Rosenberg, A.J.W.P.; Van Es, R.J.J. Resection of early oral squamous cell carcinoma with positive or close margins: Relevance of adjuvant treatment in relation to local recurrence: Margins of $3 \mathrm{~mm}$ as safe as $5 \mathrm{~mm}$. Oral Oncol. 2014. [CrossRef] [PubMed]

5. Zabrodsky, M.; Lukes, P.; Lukesova, E.; Boucek, J.; Plzak, J. The Role of Narrow Band Imaging in the Detection of Recurrent Laryngeal and Hypopharyngeal Cancer after Curative Radiotherapy. Biomed Res. Int. 2014. [CrossRef] [PubMed]

6. Miles, B.A.; Patsias, A.; Quang, T.; Polydorides, A.D.; Richards-Kortum, R.; Sikora, A.G. Operative margin control with high-resolution optical microendoscopy for head and neck squamous cell carcinoma. Laryngoscope 2015. [CrossRef]

7. Liu, W.; Wang, Y.M.; Li, Y.H.; Cai, S.J.; Yin, X.B.; He, X.W.; Zhang, Y.K. Fluorescent Imaging-Guided Chemotherapy-and-Photodynamic Dual Therapy with Nanoscale Porphyrin Metal-Organic Framework. Small 2017. [CrossRef]

8. Bethune, G.; Bethune, D.; Ridgway, N.; Xu, Z. Epidermal growth factor receptor (EGFR) in lung cancer: An overview and update. J. Thorac. Dis. 2010, 2, 48-51.

9. Ali, R.; Wendt, M.K. The paradoxical functions of EGFR during breast cancer progression. Signal Transduct. Target. Ther. 2017, 2, 16042. [CrossRef]

10. Walker, F.; Abramowitz, L.; Benabderrahmane, D.; Duval, X.; Descatoire, V.; Hénin, D.; Lehy, T.; Aparicio, T. Growth factor receptor expression in anal squamous lesions: Modifications associated with oncogenic human papillomavirus and human immunodeficiency virus. Hum. Pathol. 2009, 40, 1517-1527. [CrossRef]

11. Xu, H.; Zong, H.; Ma, C.; Ming, X.; Shang, M.; Li, K.; He, X.; Du, H.; Cao, L. Epidermal growth factor receptor in glioblastoma. Oncol. Lett. 2017, 14, 512-516. [CrossRef] [PubMed]

12. Zimmermann, M.; Zouhair, A.; Azria, D.; Ozsahin, M. The epidermal growth factor receptor (EGFR) in head and neck cancer: Its role and treatment implications. Radiat. Oncol. 2006, 1, 11. [CrossRef] [PubMed]

13. Pola, R.; Parnica, J.; Zuska, K.; Bohmová, E.; Filipová, M.; Pechar, M.; Pankrác, J.; Mucksová, J.; Trefil, P.; Kalina, J.; et al. Oligopeptide-targeted polymer nanoprobes for fluorescence-guided endoscopic surgery. Multifunct. Mater. 2019, 2, 24004. [CrossRef]

14. Franovic, A.; Gunaratnam, L.; Smith, K.; Robert, I.; Patten, D.; Lee, S. Translational up-regulation of the EGFR by tumor hypoxia provides a nonmutational explanation for its overexpression in human cancer. Proc. Natl. Acad. Sci. USA 2007, 104, 13092-13097. [CrossRef] [PubMed]

15. Orcutt, K.P.; Parsons, A.D.; Sibenaller, Z.A.; Scarbrough, P.M.; Zhu, Y.; Sobhakumari, A.; Wilke, W.W.; Kalen, A.L.; Goswami, P.; Miller, F.J.; et al. Erlotinib-Mediated Inhibition of EGFR Signaling Induces Metabolic Oxidative Stress through NOX4. Cancer Res. 2011, 71, 3932-3940. [CrossRef] [PubMed]

16. Maeda, H. Macromolecular therapeutics in cancer treatment: The EPR effect and beyond. J. Control. Release 2012, 164, 138-144. [CrossRef] [PubMed]

17. Maeda, H.; Matsumura, Y. Tumoritropic and Lymphotropic Principles of Macromolecular Drugs. Crit. Rev. Ther. Drug Carrier Syst. 1989, 6, 193-210.

18. Etrych, T.; Mrkvan, T.; Ǩíhová, B.; Ulbrich, K. Star-shaped immunoglobulin-containing HPMA-based conjugates with doxorubicin for cancer therapy. J. Control. Release 2007, 122, 31-38. [CrossRef]

19. Etrych, T.; Strohalm, J.; Kovář, L.; Kabešová, M.; Ř́hová, B.; Ulbrich, K. HPMA copolymer conjugates with reduced anti-CD20 antibody for cell-specific drug targeting. I. Synthesis and in vitro evaluation of binding efficacy and cytostatic activity. J. Control. Release 2009, 140, 18-26. [CrossRef]

20. Etrych, T.; Strohalm, J.; Chytil, P.; Černoch, P.; Starovoytova, L.; Pechar, M.; Ulbrich, K. Biodegradable star HPMA polymer conjugates of doxorubicin for passive tumor targeting. Eur. J. Pharm. Sci. 2011, 42, 527-539. [CrossRef]

21. Imagej. Available online: https://imagej.net (accessed on 11 October 2018).

22. Li, S.; Schmitz, K.R.; Jeffrey, P.D.; Wiltzius, J.J.W.; Kussie, P.; Ferguson, K.M. Structural basis for inhibition of the epidermal growth factor receptor by cetuximab. Cancer Cell 2005, 7, 301-311. [CrossRef] [PubMed]

23. Genta, I.; Chiesa, E.; Colzani, B.; Modena, T.; Conti, B.; Dorati, R. GE11 peptide as an active targeting agent in antitumor therapy: A minireview. Pharmaceutics 2018, 10, 2. [CrossRef] [PubMed] 
24. Mazzuca, C.; Di Napoli, B.; Biscaglia, F.; Ripani, G.; Rajendran, S.; Braga, A.; Benna, C.; Mocellin, S.; Gobbo, M.; Meneghetti, M.; et al. Understanding the good and poor cell targeting activity of gold nanostructures functionalized with molecular units for the epidermal growth factor receptor. Nanoscale Adv. 2019, 1, 1970-1979. [CrossRef]

25. Etrych, T.; Subr, V.; Strohalm, J.; Sírová, M.; Ríhová, B.; Ulbrich, K. HPMA copolymer-doxorubicin conjugates: The effects of molecular weight and architecture on biodistribution and in vivo activity. J. Control. Release 2012, 164, 346-354. [CrossRef] [PubMed]

26. Seymour, L.W.; Miyamoto, Y.; Maeda, H.; Brereton, M.; Strohalm, J.; Ulbrich, K.; Duncan, R. Influence of molecular weight on passive tumour accumulation of a soluble macromolecular drug carrier. Eur. J. Cancer 1995, 31, 766-770. [CrossRef]

27. Tolmachev, V.; Orlova, A.; Wei, Q.; Bruskin, A.; Carlsson, J.; Gedda, L. Comparative Biodistribution of Potential Anti- Glioblastoma Conjugates [ 111 In]DTPA-hEGF and [ 111 In]Bz-DTPA-hEGF in Normal Mice. Cancer Biother. Radiopharm. 2004, 19, 491-502. [CrossRef]

28. Huang, L.; Gainkam, L.O.T.; Caveliers, V.; Vanhove, C.; Keyaerts, M.; De Baetselier, P.; Bossuyt, A.; Revets, H.; Lahoutte, T. SPECT Imaging with 99mTc-Labeled EGFR-Specific Nanobody for In Vivo Monitoring of EGFR Expression. Mol. Imaging Biol. 2008, 10, 167-175. [CrossRef]

29. Liu, F.; Jiao, Y.; Jiao, Y.; Garcia-Godoy, F.; Gu, W.; Liu, Q. Sex difference in EGFR pathways in mouse kidney-potential impact on the immune system. BMC Genet. 2016, 17, 146. [CrossRef]

(C) 2020 by the authors. Licensee MDPI, Basel, Switzerland. This article is an open access article distributed under the terms and conditions of the Creative Commons Attribution (CC BY) license (http://creativecommons.org/licenses/by/4.0/). 